\title{
Application of sex-specific single-nucleotide polymorphism filters in genome-wide association data
}

\author{
Hua Ling ${ }^{1}$, Kurt Hetrick ${ }^{1}$, Joan E Bailey-Wilson ${ }^{2}$ and Elizabeth W Pugh* ${ }^{1}$
}

Addresses: ${ }^{1}$ Center for Inherited Disease Research, Institute of Genetic Medicine, Johns Hopkins University School of Medicine, Baltimore, Maryland 21224, USA and ${ }^{2}$ Inherited Disease Research Branch, National Human Genome Research Institute, National Institutes of Health, Baltimore, Maryland 21224, USA

E-mail: Hua Ling - hling1@jhmi.edu; Kurt Hetrick - khetric1@jhmi.edu; Joan E Bailey-Wilson - jebw@mail.nih.gov; Elizabeth W Pugh* - epugh1@jhmi.edu

${ }^{*}$ Corresponding author

from Genetic Analysis Workshop 16

St Louis, MO, USA 17-20 September 2009

Published: 15 December 2009

BMC Proceedings 2009, 3(Suppl 7):S57 doi: 10.1186/I753-656I-3-S7-S57

This article is available from: http://www.biomedcentral.com/I753-656I/3/S7/S57

(C) 2009 Ling et al; licensee BioMed Central Ltd.

This is an open access article distributed under the terms of the Creative Commons Attribution License (http://creativecommons.org/licenses/by/2.0), which permits unrestricted use, distribution, and reproduction in any medium, provided the original work is properly cited.

\begin{abstract}
We explored five sex-specific quality control filters in North American Rheumatoid Arthritis Consortium's Illumina $550 \mathrm{k}$ datasets. Three $\mathrm{X}$ chromosome and three autosomal single-nucleotide polymorphisms flagged by sex quality control filters were missed by filters of call rate at $95 \%$ and Hardy-Weinberg equilibrium at $10^{-6}$. We applied a subset of these sex-specific quality control filters to eight chromosomes in the Framingham Heart Study samples genotyped by Affymetrix $500 \mathrm{k} \mathrm{SNP}$ arrays, and identified another two single-nucleotide polymorphisms that failed to be picked up by the above global filters.
\end{abstract}

\section{Background}

The advent of genome-wide association study (GWAS) arrays with hundreds of thousands of markers relegates manual review of genotyping data to, at best, a small subset of the markers, which often are the single-nucleotide polymorphisms (SNPs) of interest after analysis. SNP filters such as call frequency, minor allele frequency (MAF), Hardy-Weinberg equilibrium (HWE), replicate concordance, and mendelian errors have been widely implemented to identify problematic SNPs. For example, the SNP thresholds used by Plenge et al. [1] for the rheumatoid arthritis (RA) study that includes the North American Rheumatoid Arthritis Consortium (NARAC) samples were call frequency $>95 \%, \mathrm{MAF}>1 \%$, and HWE $p$-value $>10^{-6}$ (the NARAC and Swedish Epidemiologic Investigation of Rheumatoid Arthritis (EIRA) data sets were filtered separately) [1]. Such global SNP filters are effective in removing SNPs with clustering problems, thus reducing the large number of highly significant associations, and lowering genomic control value lambda toward expectation. However, manual review of SNP plots suggests that some good SNPs are deleted and some bad ones remain. In particular, we have observed SNPs on the X chromosome for which the male and female samples cluster differently on Illumina GWAS arrays. The raw and normalized intensity plots for these SNPs exhibit different clusters by sex. Calls for one sex may be missing or assigned to an incorrect genotype. Also, Illumina's Beadstudio calling 
algorithm ignores sex in clustering, and will make illogical calls, e.g., heterozygous calls for males on the $\mathrm{X}$ chromosome or genotype calls for females on the $\mathrm{Y}$ chromosome. We have noted sex differences for a small number of pseudo-autosomal and autosomal markers as well.

Such SNPs, where data is differentially missing, miscalled, or both by sex may be of particular concern for diseases with differences in risk by sex, such as RA. In this analysis, we report on sex-specific differences in SNP call rates and genotype frequencies in the NARAC and Framingham Heart Study (FHS) data as released in Genetic Analysis Workshop 16 (GAW16).

\section{Methods}

Data were extracted from NARAC and FHS data files for GAW16 and analyzed using PLINK 1.04 [2]. In the NARAC dataset, DNA samples from 2,062 individuals (868 cases and 1,194 controls, 569 males and 1493 females) were genotyped on Illumina $550 \mathrm{k}$ arrays. For the FHS dataset, 6,848 individuals were genotyped on Affymetrix GeneChip ${ }^{\circledR}$ Human Mapping 500 k Array Set. We used the 6,808 of FHS samples with a specified sex $(3,105$ males and 3,703 females).

The overall sample and SNP statistics including observed proportion of heterozygotes (PropHet), call rate, and MAF were calculated using PLINK. We explored three approaches to identifying $\mathrm{X}$ chromosome SNPs that may be called differently by sex. The simplest were the proportion of male heterozygote calls and the absolute value of the difference in the call rates for males and females. The other, more statistical approach was to code all samples as female (because PLINK drops male heterozygote genotypes on $\mathrm{X}$ ), use sex as the phenotype, and test whether the proportion of missing data was associated with sex (-test-missing option in PLINK [2]). We used four approaches for autosomes: the absolute value of the differences in the call rates and proportion of heterozygotes in all samples, the test of missing data by sex, and a test of allelic association with sex among the controls (-test-missing and -assoc option in PLINK [2]). SAS and Spotfire DecisionSite 9.1.1 [3] were used for additional calculations and for plots. For SNPs with extreme difference in proportion of heterozygotes, we used the BLASTn program to search for sequence alignments using the surrounding sequence of the SNP as provided by dbSNP. Eight of the autosomes with SNPs flagged for sex discrepancy in the NARAC data were further examined in the FHS dataset using the same approaches.

\section{Results}

\section{NARAC chromosome $X$}

The call rates for males and females are shown in Figure 1a. Figure $1 \mathrm{~b}$ plots the overall call rate and the proportion of male heterozygotes. Ideally, one might examine a subset of plots from various portions of the distribution of each QC metric and attempt to choose a threshold beyond which most of the plots confirm a clustering problem. Lacking the intensity data, we chose a call rate difference corresponding to the amount of overall data we would accept for a SNP. For instance, a $5 \%$ call rate difference corresponding to a 95\% call rate (289 SNPs). There should be no male heterozygote calls. We chose a threshold to flag more than a small number of errant calls given our sample size of 569 males: a $1 \%$ threshold flags 76 SNPs. For the missingness test there are 345 SNPs with $p$-values less than $10^{-7}$. This method flags SNPs with smaller differences between males and females when the overall call rate for the SNP is higher. Figure 1c plots the overall call rate by sex difference in call rate for chromosome $\mathrm{X}$, and highlights in red the SNPs with $p$-values less than $10^{-7}$.

There are 151, 248, and 21 SNPs flagged exclusively by one, two, or three of these flags. After applying other QC filters of an overall call rate higher than $95 \%$ and a

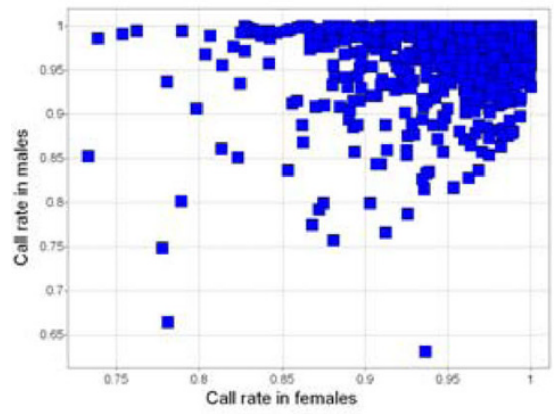

b

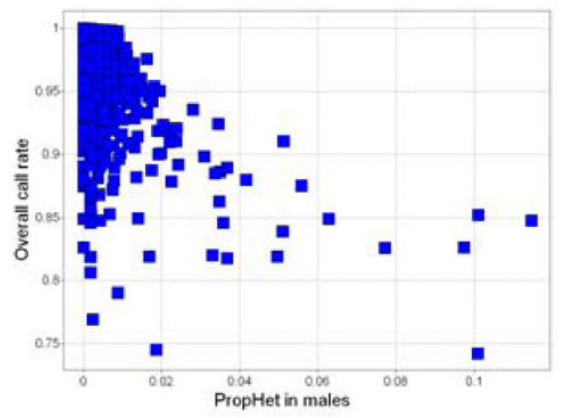

C

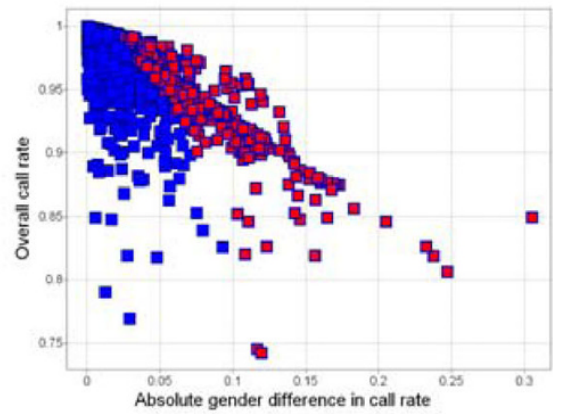

Figure I

Plots of sex-specific QC metrics for chromosome X SNPs. a, Call rate in males vs. females; b, Overall call rate vs. proportion of male heterozygote calls; c, Overall call rate vs. absolute sex difference in call rate. 
Table I: Chromosome X SNPs flagged by three sex-specific QC metrics

\begin{tabular}{|c|c|c|c|c|c|c|}
\hline SNP & Position & Call rate $(\%)$ & HWE $p$ in female controls & $\begin{array}{c}\text { Abs diff } \\
\text { call rate (\%) }\end{array}$ & $\begin{array}{c}\text { Proportion male } \\
\text { heterozygotes (\%) }\end{array}$ & Missing test by sex \\
\hline $\begin{array}{l}\text { rs4893596 } \\
\text { rs705I891 } \\
\text { rs7884I74 }\end{array}$ & $\begin{array}{l}2.9 \times 10^{7} \\
2.9 \times 10^{7} \\
6.4 \times 10^{7}\end{array}$ & $\begin{array}{c}95 \\
95.49 \\
95.83\end{array}$ & $\begin{array}{c}0.144 \\
1 \\
0.033\end{array}$ & $\begin{array}{c}7.36 \\
1.1 \\
9.45\end{array}$ & $\begin{array}{l}1.95 \\
1.4 \\
1.18\end{array}$ & $\begin{array}{l}1.69 \times 10^{-11} \\
3.50 \times 10^{-25} \\
3.26 \times 10^{-20}\end{array}$ \\
\hline
\end{tabular}

Hardy-Weinberg $p$-values among controls greater than $10^{-6}$, there are 108,90 , and 3 of the SNPs that passed these tests had one, two, or three of the sex-difference flags. The three SNPs flagged by all three methods are shown in Table 1.

\section{NARAC autosomes}

Figure 2 a plots the sex-specific call rates for the autosomal SNPs. For 94 SNPs the absolute values of the call rate difference is greater than $5 \%$. Figure $2 \mathrm{~b}$ plots the proportion of heterozygotes for males and females. In this case, we chose a threshold that would identify the outliers in these data. The six outlier SNPs can be flagged with a difference in the proportion of heterozygotes greater than $13 \%$ (highlighted in red). Three are extreme outliers (circled in Figure 2b). These three SNPs are the only SNPs having a sex association more significant than $10^{-7}$. By utilizing the surrounding sequence for these three SNPs as provided by dbSNP, these three show significant alignment to both chromosomes 1 and $\mathrm{Y}$, resulting in very similar BLASTn E-values and alignment scores [4]. Twenty-three SNPs have a $p$-value less than $10^{-7}$ for the test of missingness by sex. There are 96 SNPs flagged by one or more of these thresholds $(75,15,3$,

a

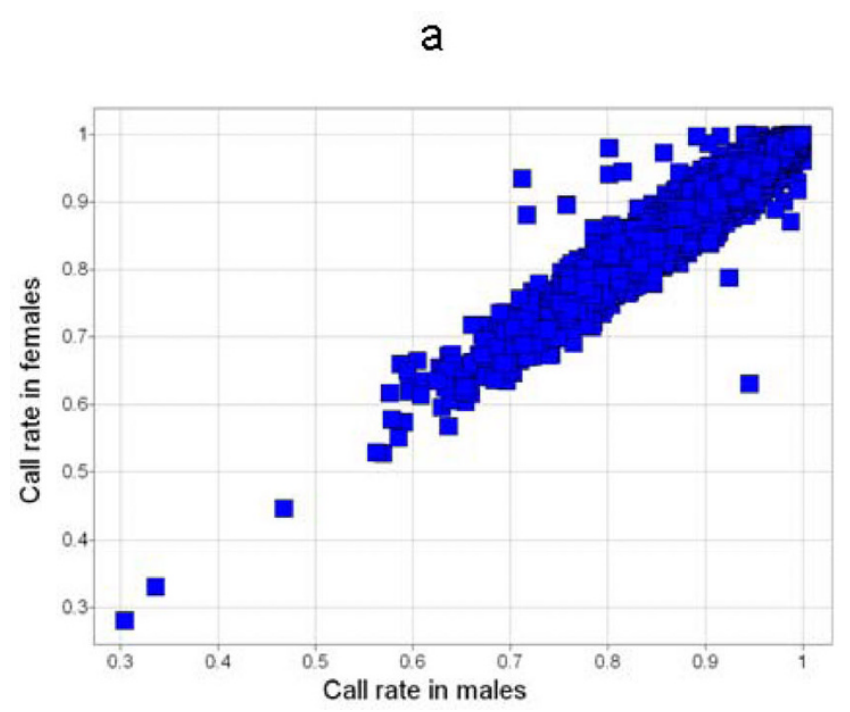

and 3 by one, two, three, and four, respectively). Table 2 lists the 21 SNPs flagged by two or more criteria. After removing SNPs that would be flagged by a 95\% overall call rate filter or a HWE filter at $10^{-6}$, there are three SNPs with two sex-difference flags and three with one flag.

\section{FHS autosomes}

We also looked at sex differences on a subset of eight chromosomes from the FHS dataset that were genotyped by Affymetrix $500 \mathrm{k}$ arrays. While there were SNPs $(n=$ $51)$ with sex differences in call rate (>5\%) and/or PropHet $(>10 \%)$ on the chromosomes we examined (Figure 2), all but two of these SNPs would also have been flagged by a $95 \%$ call rate or $10^{-6}$ HWE filter.

\section{Discussion}

Based on our experience in calling X chromosome data on the Illumina platforms, we expected to find a subset of SNPs where the male-female intensity differences shifted the clusters enough that there would be differences in call rate or some males would be called as heterozygotes. There were outliers for these measures and a test for differential missingness does flag SNPs in the NARAC data that may represent clustering problems.

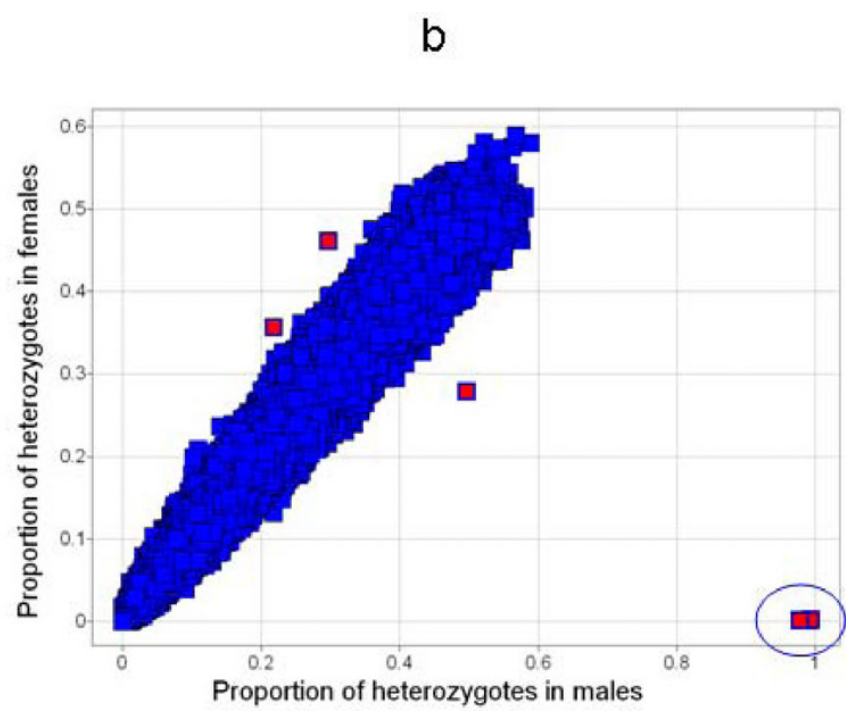

Figure 2

Plots of sex-specific QC metrics for autosomal SNPs. a, Call rate in females vs. males; b, Proportion of heterozygotes in females vs. males. 
Table 2: Autosomal SNPs flagged by at least two sex-specific QC metrics

\begin{tabular}{|c|c|c|c|c|c|c|c|c|}
\hline SNP & Chr & Position & Call rate $(\%)$ & $\begin{array}{l}\text { HWE } p \text { in } \\
\text { controls }\end{array}$ & $\begin{array}{l}\text { Abs diff call } \\
\text { rate }(\%)\end{array}$ & $\begin{array}{l}\text { Abs diff in Het } \\
(\%)\end{array}$ & $\begin{array}{l}\text { Missing test } \\
\text { by sex }\end{array}$ & $\begin{array}{l}\text { Sex association } \\
\text { in controls }\end{array}$ \\
\hline rs 12734338 & 1 & 199201380 & 98.3 & $2.76 \times 10^{-12}$ & 5.91 & 98.87 & $1.22 \times 10^{-18}$ & $4.86 \times 10^{-212}$ \\
\hline rs 12743401 & I & 199208305 & 98.4 & $1.32 \times 10^{-11}$ & 5.8 & 98.98 & $1.77 \times 10^{-19}$ & $7.36 \times 10^{-212}$ \\
\hline rs388I953 & i & 199259678 & 97.48 & $2.29 \times 10^{-11}$ & 8.17 & 97.5 & $2.74 \times 10^{-23}$ & $1.16 \times 10^{-207}$ \\
\hline rs6697552 & i & 239452393 & 96.41 & 0.097 & 8.39 & 9.28 & $2.09 \times 10^{-17}$ & 0.009 \\
\hline rs7584345 & 2 & $6762994 \mid$ & 91.22 & 0.389 & 8.24 & 3.88 & $1.57 \times 10^{-10}$ & 0.007 \\
\hline rs689662 & 3 & 16389202 & 96.8 & 0.668 & 10.63 & 2.14 & $3.93 \times 10^{-31}$ & 0.005 \\
\hline rs 17042252 & 3 & 16435774 & 95.15 & 0.192 & 5.48 & 5.08 & $6.40 \times 10^{-09}$ & 0.18 \\
\hline rs/2508842 & 4 & 92374678 & 90.25 & 0.068 & 13.97 & 11.58 & $2.69 \times 10^{-19}$ & 0.005 \\
\hline rs 11739167 & 5 & 25945521 & 71.82 & $1.89 \times 10^{-6}$ & 31.39 & 21.74 & $4.16 \times 10^{-55}$ & 0.157 \\
\hline rsllI55996 & 6 & 155056263 & 85.79 & $5.30 \times 10^{-11}$ & 13.87 & 10.42 & $1.58 \times 10^{-14}$ & 0.003 \\
\hline rs 10277011 & 7 & 64350823 & 94.81 & 0.051 & 5.47 & 6.75 & $4.26 \times 10^{-08}$ & 0.249 \\
\hline rs6989593 & 8 & 47670421 & 83.61 & $1.53 \times 10^{-7}$ & 16.44 & 13.83 & $7.38 \times 10^{-18}$ & 0.599 \\
\hline rs2309966 & 9 & 71344898 & 90.93 & 0.052 & 12.96 & 6.61 & $5.89 \times 10^{-18}$ & 0.873 \\
\hline rs7046290 & 9 & 87432131 & 93.94 & I & 7.64 & 1.08 & $3.50 \times 10^{-14}$ & 0.785 \\
\hline rsl1034170 & II & 4819429 & 82.59 & $4.96 \times 10^{-10}$ & 13.61 & 4.64 & $1.32 \times 10^{-14}$ & 0.63 \\
\hline rs7/24728 & 11 & 70554083 & 93.06 & 0.855 & 17.85 & 8.24 & $2.16 \times 10^{-40}$ & 0.02 \\
\hline rs703842 & 12 & 56449006 & 94.71 & 0.143 & 6.57 & 1.81 & $4.93 \times 10^{-12}$ & 0.211 \\
\hline rs 17768343 & 14 & 37070932 & 87.34 & $1.40 \times 10^{-4}$ & 22.33 & 16.5 & $1.30 \times 10^{-37}$ & 0.176 \\
\hline rs 2236225 & 14 & 63978598 & 90.35 & $4.81 \times 10^{-4}$ & 11.63 & 8.9 & $3.12 \times 10^{-20}$ & 0.142 \\
\hline rs 7294 & 16 & 31009822 & 94.13 & 0.02 & 11.56 & 5.25 & $1.38 \times 10^{-20}$ & 0.132 \\
\hline rs6503096 & 17 & 8122992 & 92.34 & 0.851 & 7.91 & 3.78 & $2.82 \times 10^{-11}$ & 0.029 \\
\hline
\end{tabular}

While the possible explanation for sex discrepancies on autosomal SNPs is less clear, we did see a small number of autosomal SNPs as outliers for our sex-specific metrics in the NARAC and FHS data. Most of these are also identified by more traditional QC filters. But a small number would not have been flagged by overall call rate or HWE. Adding sex-specific metrics to the SNP statistics for GWAS studies may allow the original and later investigators to define thresholds to flag these SNPs so that they could be dropped before, or scrutinized more carefully after, analysis. For the tables in the paper we highlighted SNPs with multiple problems. Any one flag may be sufficient to indicate a clustering problem and a need for further review. Knowing there are sex-specific differences with a SNP could aid investigators reviewing a plot of the intensity data. Highlighting males or females while the other sex is colored by genotype can make it much easier to see a sex-specific problem.

Of the five metrics we used, two looked at missingness by sex and two looked at differences in genotype frequencies. The call rate differences by sex and test of missingness are similar, but the test of missingness is affected by the overall call rate for the SNP. Likewise the difference in the proportion of heterozygotes and the test of association with sex look for sex-specific differences in genotype frequencies, with the association test flagging smaller differences when the MAF is low. When the trait is associated with sex, extra care must be taken in applying and interpreting the results of sex-specific filters. Because sex-based confounding is likely to cause at least small differences in allele and genotype frequencies, applying some checks only in the controls may be helpful.

\section{Conclusion}

In the NARAC dataset, we flagged three SNPs on the X chromosome and three on autosomes with sex-specific calling errors that would not have been detected with general QC filters (95\% call rate and $10^{-6} \mathrm{HWE}$ ). We flagged two such SNPs in the eight FHS autosomes we examined. Applied with care, sex-specific filters may be useful to identify and filter SNPs before the analysis of GWAS data.

\section{List of abbreviations used}

EIRA: Epidemiologic Investigation of Rheumatoid Arthritis; FHS: Framingham Heart Study; GAW16: Genetic Analysis Workshop 16; GWAS: Genome-wide association study; HWE: Hardy-Weinberg equilibrium; MAF: Minor allele frequency; NARAC: North American Rheumatoid Arthritis Consortium; PropHet: Proportion of heterozygotes; RA: Rheumatoid arthritis; QC: Quality control; SNP: Single-nucleotide polymorphism.

\section{Competing interests}

The authors declare that they have no competing interests.

\section{Authors' contributions}

JEBW analyzed the FHS data. EWP proposed the study, drafted and critically revised the manuscript and suggested further analysis. HL analyzed the NARAC data and drafted the manuscript. $\mathrm{KH}$ reviewed results 
and suggested further analyses. All authors read and approved the final manuscript.

\section{Acknowledgements}

The Genetic Analysis Workshops are supported by NIH grant ROI GM03 1575 from the National Institute of General Medical Sciences. JEBW is supported by the intramural program of the National Human Genome Research Institute, National Institutes of Health. HL, KH, and EWP were supported by the Center for Inherited Disease Research (CIDR). CIDR is fully funded through a federal contract from the National Institutes of Health to The Johns Hopkins University, contract number HHSN268200782096C.

This article has been published as part of BMC Proceedings Volume 3 Supplement 7, 2009: Genetic Analysis Workshop 16. The full contents of the supplement are available online at http://www.biomedcentral.com/ $\underline{1753-6561 / 3 \text { ? issue }=\text { S7. }}$.

\section{References}

I. Plenge RM, Seielstad M, Padyukov L, Lee AT, Remmers EF, Ding B, Liew A, Khalili H, Chandrasekaran A, Davies LR, Li W, Tan AK, Bonnard C, Ong RT, Thalamuthu A, Pettersson S, Liu C, Tian C, Chen WV, Carulli JP, Beckman EM, Altshuler D, Alfredsson L, Criswell LA, Amos Cl, Seldin MF, Kastner DL, Klareskog L and Gregersen PK: TRAFI-C5 as a risk locus for rheumatoid arthritis-a genomewide study. N Eng J Med 2007, 357:1199-1209.

2. Purcell S, Neale B, Todd-Brown K, Thomas L, Ferreira MA, Bender D, Maller J, Sklar P, de Bakker PI, Daly MJ and Sham PC: PLINK: a tool set for whole-genome association and population-based linkage analyses. Am J Hum Genet 2007, 8I:559-575.

3. Spotfire DecisionSite 9.1.I. http://spotfire.tibco.com/products/ decisionsite.cfm.

4. Altschul SF, Madden TL, Schaffer AA, Zhang J, Zhang Z, Miller W and Lipman DJ: Gapped BLAST and PSI-BLAST: a new generation of protein database search programs. Nucleic Acids Res 1997, 25:3389-3402.

\section{Publish with Bio Med Central and every scientist can read your work free of charge}

"BioMed Central will be the most significant development for disseminating the results of biomedical research in our lifetime."

Sir Paul Nurse, Cancer Research UK

Your research papers will be:

- available free of charge to the entire biomedical community

- peer reviewed and published immediately upon acceptance

- cited in PubMed and archived on PubMed Central

- yours - you keep the copyright
BioMedcentral 